\title{
Operon formation by insertion sequence IS3 in Escherichia coli
}

\author{
Yuki Kanai ${ }^{1}$, Saburo Tsuru ${ }^{2, \otimes}$, and Chikara Furusawa ${ }^{2,3, \bigotimes}$ \\ ${ }^{1}$ Department of Biological Sciences, Graduate School of Science, The University of Tokyo, 7-3-1 Hongo, Bunkyo-ku, Tokyo, 113-0033, Japan \\ ${ }^{2}$ Universal Biology Institute, Graduate School of Science, The University of Tokyo, 7-3-1 Hongo, Bunkyo-ku, Tokyo, 113-0033, Japan \\ ${ }^{3}$ Center for Biosystem Dynamics Research, RIKEN, 6-2-3 Furuedai, Suita, Osaka, 565-0874, Japan
}

\begin{abstract}
Operons are a hallmark of the genomic and regulatory architecture of prokaryotes. However, the mechanism by which two genes placed far apart gradually come close and form operons remains to be elucidated. Here, we propose a new model of the origin of operons: Mobile genetic elements called insertion sequences can facilitate the formation of operons by consecutive insertion-deletion-excision reactions. This mechanism barely leaves traces of insertion sequences and is difficult to detect in evolution in nature. We performed, to the best of our knowledge, the first experimental demonstration of operon formation, as a proof of concept. The insertion sequence IS 3 and the insertion sequence excision enhancer are genes found in a broad range of bacterial species. We introduced these genes into insertion sequence-less Escherichia coli and found that, supporting our hypothesis, the activity of the two genes altered the expression of genes surrounding IS3, closed a 2.7 kilobase pair gap between a pair of genes, and formed new operons. This study shows how insertion sequences can facilitate the rapid formation of operons through locally increasing the structural mutation rates and highlights how coevolution with mobile elements may shape the organization of prokaryotic genomes and gene regulation.
\end{abstract}

Correspondence: tsuru@ubi.s.u-tokyo.ac.jp, chikara.furusawa@riken.jp

\section{INTRODUCTION}

Operons are clusters of genes under the control of the same promoter sequences and are a hallmark of prokaryotic genome structure (1,2) and gene regulation (3). A significant proportion of genes of the prokaryotic genome are organized into operons (4), and it is widely accepted that operons are beneficial for multiple reasons, including for better coregulation of genes (5-8).

On the other hand, the mechanism by which operons form is poorly understood (9). As understanding operon formation is fundamental to our understanding of the evolution of prokaryotes, many models for operon formation have been proposed in the last 60 years $(7,9-12)$. However, none of the proposed mechanisms of operon formation have been corroborated by experimental evidence, limiting our understanding of the types of mutations that drive them.

In principle, operons form by rearrangements, including insertions, deletions (9) (Figure 1.A), and duplications (10), but these mechanisms alone seem to be insufficient to explain the prevalence of operons in prokaryotic genomes. For instance, duplication may explain the evolution of some operons (10), but genes in operons do not necessarily have similar structures (7). Moreover, while pairs of genes can culminate into operons by random rearrangements, they are more likely to end up apart (7).

Thus, selection for gene coregulation is thought to be crucial after operon formation by the aforementioned mechanisms to account for the prevalence of operons $(5,9,13,14)$. However, while selection for coregulation can facilitate the maintenance and sophistication of operons (9), it does not facilitate their formation (7). This is because bringing two genes closer becomes adaptive only when the genes were initially clustered close enough (7). The 'selfish operon' model proposed that instead of relying on rare random rearrangements to cluster genes, operons form gradually through small intermediate steps (7). Unfortunately, this model has not found general acceptance as it fails to explain how essential genes likely form operons $(5,15)$. Operon formation likely proceeds through a mechanism that gradually ties two far apart genes (7); however, a plausible mechanism remains to be elucidated.

As genes in operons are clustered together, mechanisms that cluster functionally related genes may also facilitate the formation of operons (13). Essential genes in prokaryotic genomes seem to cluster because the genomes tend to lose tandem sequences ('persistence model' (12)); Clustering of essential genes increases the robustness of genomes against tandem deletions by making large deletions less detrimental (16). Recently, a laboratory evolution of E. coli revealed that the major cause of tandem deletions may be the activity of insertion sequences (IS, Figure 1.B) (17). This could mean that the tandem deletions by IS cluster important genes in prokaryotic genomes and may also make those genomes operon-rich.

ISs are a major cause of genetic rearrangements $(17,18)$ and therefore may as well facilitate operons to form. A recent study, based on the presence of ISs within ancient operons, inferred that ISs may form operons by facilitating rearrangements (19). However, no study seems to have associated the tandem deletion of surrounding genes by IS with the formation of operons. 
Although ISs are known to delete neighboring sequences since at least the 1970s (20-22), deletions by IS as a mechanism that promotes the formation of operons have been undervalued for the following three main reasons: (i) IS-rich genomes seem to have more operons disrupted compared to other genomes $(19,23)$. Therefore, ISs are often associated with the disruption rather than the formation of operons. (ii) IS excision is required for an operon to form by tandem deletion (Figure 1.C). However, IS excision would result in a double-strand break in the genome, which may lead to genomic degradation unless fixed by homologous recombination (24). (iii) Some of the major types of ISs rarely excise themselves (25).

The following findings have made these arguments less critical: (i) IS-rich genomes can be rich in operons (26), suggesting that IS-mediated rearrangements may not only destroy but also form operons (9). (ii) Many bacterial species have end-joining mechanisms (27). (iii) Many ISs are known to excise themselves $(28,29)$. Moreover, the excision of one of the major types of IS, IS3, that rarely excise themselves, is significantly promoted by an enzyme called IS excision enhancer (IEE) found in various bacterial species (25).

We hypothesized that, considering that IS3 is known to rapidly create deletions of various lengths adjacent to it (21, 30 ), the interaction of IS3 and IEE may promote the rapid formation of operons by IS3 deleting sequences intervening two genes and IEE excising IS3.

In this study, we assess if deletion by IS can promote the formation of operons. First, we propose a model of operon formation based on deletions by ISs, that does not rely on the selection for coregulation. We also suggest a mechanism by which an IS can facilitate the formation of operons through intermediate steps, gradually bringing closer two genes placed far apart. According to this model, ISs do not leave apparent traces after forming operons. This makes it difficult to demonstrate the model using comparative genomics of extant genomes. Therefore, as a proof of concept, we engineered $E$. coli in a state that mimics an intermediate step of the model by inserting a copy of IS3 between two conditionally essential genes. To detect the diversity of structural mutations during the formation of operons, fluorescent reporters were placed around the copy of IS3. Selecting the cells cultured overnight based on their fluorescence, we examined how the concerted activity of IS3 and IEE affects the expression of surrounding genes, leading to the formation of novel operons. We believe this study is the first to provide experimental evidence of a plausible mechanism of operon formation in prokaryotes.

\section{MATERIALS AND METHODS}

\section{Reagents}

For cell culture, we used: LB medium (Difco LB Broth, Miller, BD, USA, 244620), kanamycin (Kanamycin Sulfate, Wako, Japan, 115-00342), streptomycin (Streptomycin Sulfate, Wako, Japan, 190-14342), ampicillin
(Ampicillin Sodium, Wako, Japan, 012-23303), anhydrotetracycline hydrochloride (aTc) (Anhydrotetracycline hydrochloride, Sigma-Aldrich, USA, 37919), arabinose (L(+)Arabinose, Wako, Japan, 010-04582), IPTG (Isopropyl$\beta$-D(-)-thiogalactopyranoside [IPTG], Wako, Japan, 19014342), agar (Agar, Powder, Wako, Japan, 010-08725).

For handling nucleic acids and DNA sequencing, we used: KOD One PCR Master Mix Blue (Toyobo, Japan, KMM201), Rapid Barcoding Kit (Oxford Nanopore Technologies, UK, SQK-RBK004), DpnI (Takara Bio, Japan, 1235-A), PureLink RNA Mini Kit (Thermo Fisher Scientific, USA, 12183018A), PureLink DNase Set (Thermo Fisher Scientific, USA, 12185010), PrimeScript High Fidelity RT-PCR Kit (Takara Bio, Japan, R022A), In-Fusion Snap Assembly Master Mix (Takara Bio, Japan, 638948), FastGene Plasmid Mini Kit (NIPPON Genetics, Japan, FG-90502).

We used the following instruments: Infinite F200 multimode plate reader (Tecan, Switzerland), Flongle Flow Cell R9.4.1 (Oxford Nanopore Technologies, UK, FLO-FLG001), MinION Mk1B (Oxford Nanopore Technologies, UK, MIN101B), FACSAria III (BD, USA), MiniAmp Plus (Thermo Fisher Scientific, USA), blue/green LED transilluminator (NIPPON Genetics, Japan, LB-16BG).

The primers we used are listed in Supplementary Table 1.

\section{Biological resources}

We used the following strains: E. coli MG1655 (the origin of IS3 transposase used in this study), E. coli MDS42 (the parent strain used for laboratory evolution, (31)), E. coli HST08 (used to test coregulation of genes in newly-formed operons and for vector construction; E. coli HST08 Premium Competent Cells, Takara Bio, Japan, 9128).

We used several plasmids in this study. pCDSSara-L18 (32) was used as the backbone of pYK-1S3 and as a -iee control. The Tn5 transposase gene of pRC2117 (33) was inserted instead of IS3 transposase as a -tpn control. The following plasmids were constructed for this study: pYK-1N5 (the fluorescence reporter plasmid), pYK-1S3 (the IEE expression plasmid), pYK-1Q2 (pYK-1N5 with Tn5 transposase gene within a pair of IS3 inverted repeats), pYK-1S0 (plasmid constructed based on pYK-1N5 but with a $\mathrm{P}_{t a c}$ cassette).

\section{Strains and plasmids}

To identify the effect of IS3 on the formation of operons, $E$. coli MDS42, a derivative of the wild-type K-12 MG1655, was used as it is absent of all mobile elements, including IS3 (31). IS3 and IEE were supplied via plasmids pYK-1N5 and pYK-1S3, respectively. All plasmids were constructed using the In-Fusion cloning kit.

The IS3 supplying vector (pYK-1N5, Figure 2.A) was designed to mimic a state after the 'insertion' step of the model that we propose in this study (Figure 1.C). pYK1N5 is a low copy-number plasmid (pSC101 origin) with a kanamycin resistance gene $(\mathrm{kanR})$, an engineered IS3 sequence, and red (mScarlet-I (34)) and yellow (Venus YFP 
(35)) fluorescent protein genes $(y f p, r f p)$. To demonstrate our proposed model, IS3 was engineered to have between its two inverted repeats (IR) the IS3 transposase gene (tpn) downstream of an inducible promoter (based on $\mathrm{P}_{\text {LtetO-1 }}$ (36, 37)) and a synthetic promoter $P_{\mathrm{J} 23105}$ (a Biobrick promoter). The $\mathrm{P}_{\text {LtetO-1 }}$ derivative is repressed by the product of $t e t R$ on the same plasmid, and therefore, transcription is inducible by the addition of aTc to the growth medium. $\mathrm{P}_{\mathrm{J} 23105}$ was inserted to mimic the outward-facing promoter P3R of wildtype IS3 because, according to our model, it facilitates the formation of operons. We chose $\mathrm{P}_{\mathrm{J} 23105}$ because its strength was ideal for demonstrating using flow cytometry (FCM) the influence of IS3 activity on the expression of surrounding genes. The IS3 used in this study was designed so that the two promoters of IS 3 were divergent, with the left IR (IRL) downstream of the transposase gene. This design was chosen to avoid induction by aTc to increase the transcription of the fluorescent protein, to avoid potential promoter activity from IRL to interfere with $\mathrm{P}_{\mathrm{LtetO}-1}$, and to prevent the plasmid from being degraded before evolution by inhibiting the activity of the transposase gene by antisense transcription from the kanR gene. In addition, following a previous study (21), the two open reading frames constituting the IS3 transposase gene were fused by a single base insertion in the $A_{4} G$ motif to increase the activity of the transposase.

The IEE expression vector (pYK-1S3, Figure 2.B) was constructed based on pCDSSara-L18 (32) by replacing the arabinose-inducible ribosomal protein gene with iee of E. coli O157:H7 strain Sakai (NCBI Gene ID: 912859, ECs_1305). This plasmid had a streptomycin resistance gene $(s m R)$ as a selection marker.

Derivatives of the two plasmid vectors without the IS3 and IEE genes were used as negative controls. A hyper-active mutant of $\operatorname{Tn} 5$ transposase gene (33) and ribosomal subunit L18 (32) were placed instead of IS3 transposase and IEE genes, respectively.

To introduce IS3 and IEE, the ancestor strains for evolution were prepared by two rounds of electrotransformation. First, pYK-1S3 (or its -iee derivative) was introduced by transformation, and the transformed cells were selected on LB agar plates containing streptomycin. Mixtures of electrocompetent cells were then prepared from the selected colonies. Finally, pYK-1N5 (or its $-t p n$ derivative) was introduced by transformation into the cells and the transformed cells were grown on LB agar plates containing streptomycin and kanamycin.

\section{Culture conditions}

Cells were cultured aerobically in $200 \mu \mathrm{L}$ volume of LB medium in 96-well plates (Greiner Bio-One, Cellstar, 655180). First, the medium was filtered through a $0.2 \mu \mathrm{m}$ membrane filter (Thermofisher Scientific, 567-0020) to remove debris that may interfere with FCM measurements. The culture medium was supplemented with $50 \mu \mathrm{g} / \mathrm{mL}$ kanamycin and $100 \mu \mathrm{g} / \mathrm{mL}$ streptomycin where indicated, to select only cells with both pairs of plasmids. To induce transcription of transposase and IEE genes, $50 \mathrm{nM}$ of aTc and $0.1 \%(\mathrm{w} / \mathrm{v})$ of arabinose, respectively, were added to the media where indicated.

Growth rates were measured in Infinite F200 multimode plate reader at $37^{\circ} \mathrm{C}$ overnight. The plate was shaken linearly and then orbitally for three minutes each, and $\mathrm{OD}_{600}$ was measured every ten minutes to determine the growth rate.

To obtain colonies, cells were plated on $1.5 \%$ agar plates supplemented with LB and appropriate antibiotics.

Cells were temporarily stored at $4{ }^{\circ} \mathrm{C}$ as colonies grown on LB agar plates or as mixtures in filtered phosphate-buffered saline (PBS).

\section{Flow cytometry and selection of cells by fluorescence- activated cell sorting (FACS)}

For the evolution of the cells, four colonies of each of the four genotypes ( \pm tpn and \pm iee) were picked with toothpicks from LB agar plates, diluted 100-fold, and cultured overnight in 96-well plates with $200 \mu \mathrm{L} \mathrm{LB}$ containing streptomycin and kanamycin supplemented with aTc and arabinose. After overnight culture at $30^{\circ} \mathrm{C}$, the cells were diluted in ice-cold PBS and stored at $4{ }^{\circ} \mathrm{C}$ until FCM measurements. The singlecell fluorescence of these cells was measured by FCM using FACSAria III and FACS Diva software v.6.1.3. The analysis and visualization were based on single-cell fluorescence of cells with forward scatter measurements within a twofold range, including the most frequent values to compare fluorescence among cells in similar physiological states (38).

For FACS, four populations of cells with both tpn and iee and one population of cells with tpn but without iee were prepared as described above. After a brief measurement of the distribution of fluorescence by FCM using 10,000 cells, gates were manually set by first subsetting the measurements with the same forward scattered values as above and then manually drawing the gates according to the measurement, as shown in Figure 4.A (P1-3). The lines of the gates were made thicker for visualization using Inkscape. The same gates were used for all five rounds of FACS. The cells were collected in $500 \mu \mathrm{L}$ of $\mathrm{LB}$ or PBS.

\section{Genotyping cells}

To confirm that the cells were sorted as expected, 200-500 $\mu \mathrm{L}$ of the collected cells were cultured overnight in $5 \mathrm{~mL}$ of LB medium containing kanamycin and streptomycin, and $10 \mu \mathrm{L}$ of cultured cells were plated on LB agar plates with kanamycin and streptomycin. The plates were incubated at $37^{\circ} \mathrm{C}$ for two days and imaged under a transilluminator. The brightness and contrast were adjusted using ImageJ (National Institutes of Health, USA).

Sanger sequencing was used to genotype the cells in each FACS gate. Colonies on agar plates showing uniform fluorescence were picked, and colony PCR was performed using KOD One PCR Master Mix Blue. Template DNA was prepared by picking the colonies by toothpicks into Tris-HCl (pH8.5) with $0.1 \%$ Triton X-100, boiling at 98 
${ }^{\circ} \mathrm{C}$ for five minutes, and spinning down briefly at 10,000 $\times \mathrm{g}$. The supernatants were used as templates. Agarose gel electrophoresis was run for the amplified DNA, and fragments of typical lengths were chosen and sequenced by Sanger sequencing (GeneWiz). The same procedure, but with a different primer set, was followed to check that sequences were not deleted in plasmids of cells in the major dark fraction of FCM measurements in +iee +tpn cells.

To identify the diversity among the cells sorted by FACS, nanopore sequencing was performed. $400 \mu \mathrm{L}$ out of 500 $\mu \mathrm{L}$ of PBS with 600 cells collected by a single round of FACS were transferred into $5 \mathrm{~mL}$ of LB medium containing kanamycin and cultured overnight at $37^{\circ} \mathrm{C}$. Streptomycin was not added to reduce the read mapping onto pYK-1S3 and increase read mapping onto pYK-1N5. Plasmid DNA was extracted with FastGene Plasmid Mini Kit (with optional washing), and the sample for sequencing was prepared by adding barcodes and adapters using Rapid Barcoding Kit following the manufacturer's instructions. The samples were applied to Flongle Flow Cell R9.4.1 on MinION Mk1B and sequenced using MinKNOW (v21.06.0). The fast5 data was basecalled using ONT Guppy (v5.0.11) with the configuration set to the 'superaccurate model.' The reads were filtered according to the default quality threshold, and the barcode sequences were trimmed. We obtained 3,522 reads with $14,705,564$ base pairs (bp) corresponding to a sequencing depth of approximately 2,000. The median read length and quality were $4,359 \mathrm{bp}$ and 13.03 , respectively. Reads longer than one kbp were filtered with Filtlong (v0.2.0, https://github.com/rrwick/Filtlong) and mapped onto a fasta file with two copies of the pYK-1N5 sequence placed in tandem using minimap2 (v2.17-r941 (39)) to avoid reads that were mapped across the origin being split. Deletions longer than $50 \mathrm{bp}$ were detected and extracted from the CIGAR strings of BAM using Rsamtools (v2.6.0) and analyzed using a custom script. The 514 reads are plotted in Figure 4.F.

\section{Reverse transcription polymerase chain reaction (RT- PCR)}

To assess if kanR and $r f p$ formed an operon, RT-PCR was performed. Two colonies genotyped by Sanger sequencing were picked into LB medium containing streptomycin and kanamycin and cultured overnight at $30^{\circ} \mathrm{C}$. The cultured cells were diluted 50-fold into a fresh growth medium and cultured at $30{ }^{\circ} \mathrm{C}$ for two hours. The total RNA was extracted using the PureLink RNA Mini Kit. DNA was removed using DNAse during the extraction. Total RNA was amplified using PrimeScript High Fidelity RT-PCR Kit following the manufacturer's two-step protocol. First, reverse transcription was performed. RNA with a final concentration of approximately $50 \mathrm{ng} / \mu \mathrm{L}$ was denatured at $65{ }^{\circ} \mathrm{C}$ for five minutes with either of the forward and reverse primers and, as a negative control of reverse transcription, with neither of the primers; reverse-transcriptase and buffers were added on ice; reverse transcription was performed at $42{ }^{\circ} \mathrm{C}$ for 30 minutes; the reverse transcriptase was denatured at $95^{\circ} \mathrm{C}$ for five minutes; the RNA mixtures were stored at $4{ }^{\circ} \mathrm{C}$.
Then, cDNA was amplified by PCR. The obtained cDNA was diluted ten-fold with a premix containing the pair of primers and the DNA polymerase; DNA was amplified by 20 cycles of PCR (MiniAmp Plus), with denaturation at $98^{\circ} \mathrm{C}$ for ten seconds, followed by annealing at $57^{\circ} \mathrm{C}$ for five seconds, and extension at $72{ }^{\circ} \mathrm{C}$ for three minutes. The PCR products were subject to gel electrophoresis, and the image was recorded under a transilluminator. The brightness and contrast of the image were adjusted using ImageJ.

\section{Testing the coregulation of kanR and $r f p$ in newly- formed operons}

To further confirm operon formation after evolution, the kanR promoter of pYK-1N5 and its evolved derivatives were replaced with a lactose inducible promoter $\mathrm{P}_{t a c}$. DNA sequences, excluding the kanamycin promoter sequence, of the plasmids were amplified by PCR (KOD One PCR Master Mix Blue). Another sequence containing the ampicillin resistance gene, $\mathrm{P}_{t a c}$, and lacI (Supplementary Figure 6) was amplified by PCR from plasmid pYK-1S0. After digestion of remnant plasmids with DpnI, the amplified sequences were fused with In-Fusion cloning following the manufacturer's manual, and the DNA mixture was introduced to E. coli HST08 Premium Competent Cells. The sequence of the insert is provided in the DNA Sequences section of Supplementary Data.

Cells with the re-engineered plasmids were spread on LB agar plates with ampicillin. After checking the genotypes of the cells, their colonies were picked and transferred to 96well plates with $200 \mu \mathrm{L}$ of LB supplemented with ampicillin and $30 \mu \mathrm{M}$ of IPTG. The cells were cultured overnight at $32{ }^{\circ} \mathrm{C}$ to prevent edge effects. After the cells reached the stationary phase, the cells were diluted in ice-cold PBS, and single-cell fluorescence was measured using FCM.

\section{Statistical Analyses}

Type III ANOVA was performed using FCM measurements of 16 independent cell cultures consisting of four colonies for each of the four genotypes. Because there were three treatments, there were 12 degrees of freedom within groups.

For calculating the number of cells in the three gates used for FACS, the mean and $95 \%$ confidence interval (CI) of $\log _{10}$ probability are presented $(n=4)$, assuming the normality of the distributions.

To show that IPTG induction increased red fluorescence, three independent cell cultures were prepared for each condition and genotype, and the intensity of single-cell red fluorescence was measured with FCM. First, the FCM data was subsetted based on their forward scatter values as above. Using the values of median $\log _{10}$ red fluorescence of over 10,000 measurements per culture, we performed two-sided t-tests adjusted with the Bonferroni method.

\section{Data Availability/Sequence Data Resources}

Data related to nanopore sequencing and its analysis, including the fast 5 data, reference fasta, and BAM files, 

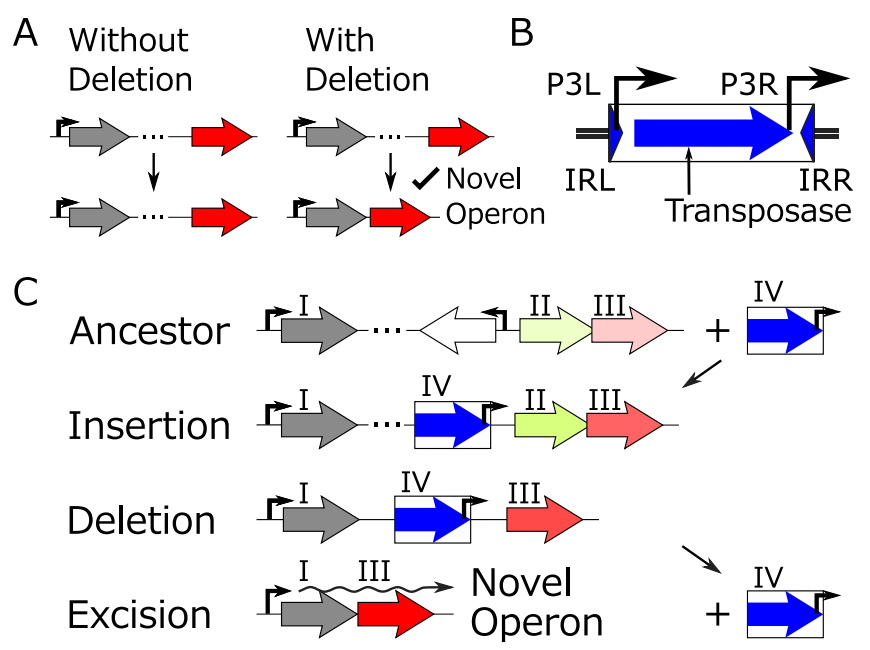

Figure 1. Insertion sequences (ISs) can facilitate the formation of novel operons through insertion-deletion-excision reactions (IDE model). (A) Deletion facilitates the formation of operons. (B) IS3 has two promoters, P3L and P3R, in the same direction. (C) An example of how ISs (IV) can facilitate the formation of operons based on the IDE model. The block arrows indicate coding sequences, and the intensity of green (II) and red (III) colors indicates the gene expression level. In an environment where the genes indicated in red (III) and gray (I) are essential and that in green (II) is nonessential, the reactions from the top to bottom leads to the genes in gray and red to form a novel operon.

were deposited in NCBI Sequence Read Archive (BioProject PRJNA768397). The raw flow cytometry data are available in FlowRepository (FR-FCM-Z4LV). DNA sequences of pYK$1 \mathrm{~N} 5$, pYK-1S3, and the $\mathrm{P}_{t a c}$ cassette are provided in the DNA sequences section of Supplementary Data.

\section{Data Availability/Novel Programs, Software, Algo- rithms}

Not applicable.

\section{Web Sites/Data Base Referencing}

As references, we used NCBI Gene for the DNA sequence of iee, and the Registry of Standard Biological Parts to find various Biobrick promoter sequences, including $\mathrm{P}_{\mathrm{J} 23105}$.

\section{RESULTS}

\section{A model of operon formation based on the activity of insertion sequences}

To explain the formation of operons in prokaryotes, we propose a model that relies on the activity of ISs and experimentally demonstrate the formation of an operon. ISs may be able to cluster two genes placed far apart into an operon by a sequential Insertion-Deletion-Excision reaction as follows (IDE model, Figure 1.C).

Ancestor: Initially, two genes beneficial in the same environment are placed apart, as is the case for the genes in gray and red (I, III) in Figure 1.C.

Insertion: Many ISs have promoters that facilitate the transcription of downstream genes $(40,41)$. For example, in addition to the promoter upstream of the transposase gene, IS3 has a strong promoter facing outward (P3R, Figure 1.B
(42)). These promoters help an IS (IV) to activate initially dormant genes (II, III) by transposing upstream of the genes (41).

Deletion: Many types of ISs are known to delete sequences adjacent to them (20-22). Deletion can continue until nonessential genes between the IS and beneficial genes are deleted. This results in bringing together two beneficial genes on either side of the IS closer.

Excision: Many ISs can excise themselves, especially in the presence of IEE (25). This excision of an IS between two beneficial genes can lead to them forming a novel operon.

Thus, with the tandem deletion caused by IS, selection for the preservation of two genes (I and III) can lead to the formation of operons.

\section{The activity of IS can gradually form an operon through intermediate adaptive steps}

Mechanisms that gradually bring closer two genes seem to be more effective than rare rearrangements that directly cluster two genes to form an operon (7). Our model involves such a mechanism and can be broken down into small intermediate steps, all of which are adaptive (Figure 1.C). For example, let us assume that the stronger expression of genes indicated in gray (I) and red (III) is beneficial and that initially, the gene in gray is upregulated, but the gene in red lacks an effective promoter (ancestor). The insertion of an IS (IV) upstream of the gene in red would increase the fitness, as the gene would be transcribed from the promoters within the IS. Further activity of the IS can delete sequences around the IS, increasing the expression of the gene in red by bringing it closer to the inner promoters of the IS and gradually closing the space between the IS and the gene. Adaptive evolution can also bring the two genes closer together by increasing the robustness of cells against tandem deletions or activity of selfish elements $(12,43)$. Finally, the excision of IS would be most beneficial when cells with both gray and red genes are adjacent to the IS as it would bring the promoter of the gene in gray closer to the gene in red. The premature excision of IS is less likely to be fixed because the gene in red would lose active promoters encoded within the IS.

The IDE reactions locally accelerate rearrangements, and while they do not require the selection for better gene coregulation to form operons as long as active transcription of the two genes is maintained, adaptive evolution can facilitate operon formation by fixing intermediate steps towards forming operons (7).

\section{A fluorescence reporter system to detect deletions related to the formation of kanR-rfp operon}

To experimentally demonstrate our model of operon formation, we designed a plasmid as a model of the prokaryotic genome after the 'insertion' step (pYK-1N5, Figure 2.A), which is common in both nature and laboratory settings (30, 41, 44). Specifically, the fluorescent reporter plasmid pYK-1N5 was designed such that the kanamycin resistance gene $(k a n R$, I), a copy of IS3 (IV), and the red ( $r f p$, III) 


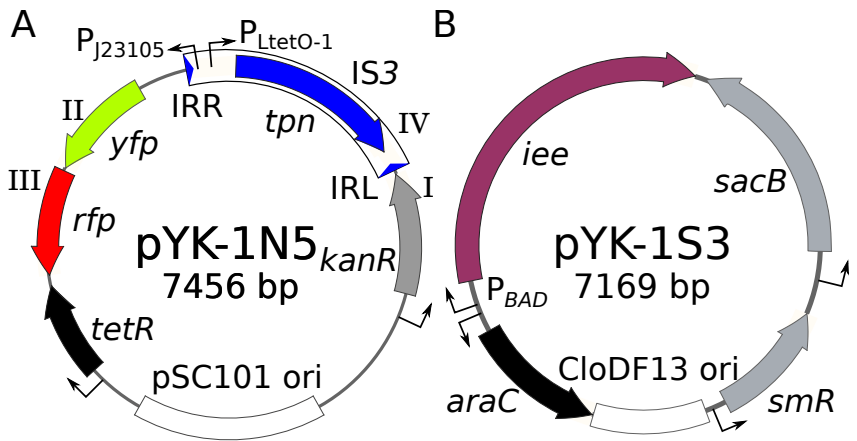

Figure 2. (A) Design of the fluorescent reporter plasmid used to detect the formation of operons. The plasmid was designed to mimic the 'insertion' step of operon formation shown in Figure 1.C. See strains and plasmids for details. (B) Design of the insertion sequence excision enhancer (IEE) expression plasmid. Tetracycline repressor gene, tet $R$; origin of replication, ori; arabinose operon regulator gene, araC; streptomycin resistance gene, smR. The genes of pYK-1N5 with roman numerals correspond to the genes in Figure 1.C with the same roman numerals.

and yellow $(y f p, \mathrm{II})$ fluorescent protein genes correspond to the genes in gray, the copy of IS, and the genes in green and red in Figure 1.C, respectively. The copy of IS3 was inserted within a $2.7 \mathrm{kbp}$ gap between $k a n R$ and $r f p$. For ease of the experiment, the wild-type IS3 was modified such that its transposase gene (tpn) was inducible by aTc, and the promoter $\mathrm{P}_{\mathrm{J} 23105}$ activated $r f p$ instead of the native promoter of IS3. We expected that the IS would delete surrounding sequences or excise itself, changing the pattern of fluorescence measured by FCM. Using FACS, we selected cells with bright red fluorescence while adding kanamycin to the growth medium, expecting the formation of kanR-rfp operons.

With the two fluorescent protein genes, various phenotypes were expected to be found after evolution. Deletions between IS3 and $y f p$ would bring $\mathrm{P}_{\mathrm{J} 23105}$ closer to $y f p$, causing both yellow and red fluorescence to increase. Excision of IS3 would lead to the transcription of the two fluorescent protein genes from the kanR promoter instead of the weaker $\mathrm{P}_{\mathrm{J} 23105 \text {, }}$ also causing both yellow and red fluorescence to increase. In this case, we expected $k a n R-y f p-r f p$ operon, or in case of $y f p$ deletion, kanR-rfp operon to form.

\section{The pattern of fluorescence observed under the presence of both IS3 and IEE}

As a potential genetic background that facilitates operon formation, we examined whether the combined activity of IS3 and IEE in E. coli can accelerate operon formation. IS3 rarely excises itself on its own but is frequently excised under the presence of IEE (25). The presence of IEE thus is expected to promote the 'excision' step of operon formation. To test this, an arabinose-inducible IEE expression vector (pYK-1S3, Figure 2.B), in which IEE was expressed from the promoter of araBAD operon $\left(\mathrm{P}_{B A D}\right)$, was constructed. As negative controls, derivatives of pYK-1N5 and pYK-1S3 without tpn and iee, respectively, were also constructed. We transformed an IS-less strain of E. coli MDS42 (31) with pairs of plasmids with and without tpn and iee. We expected

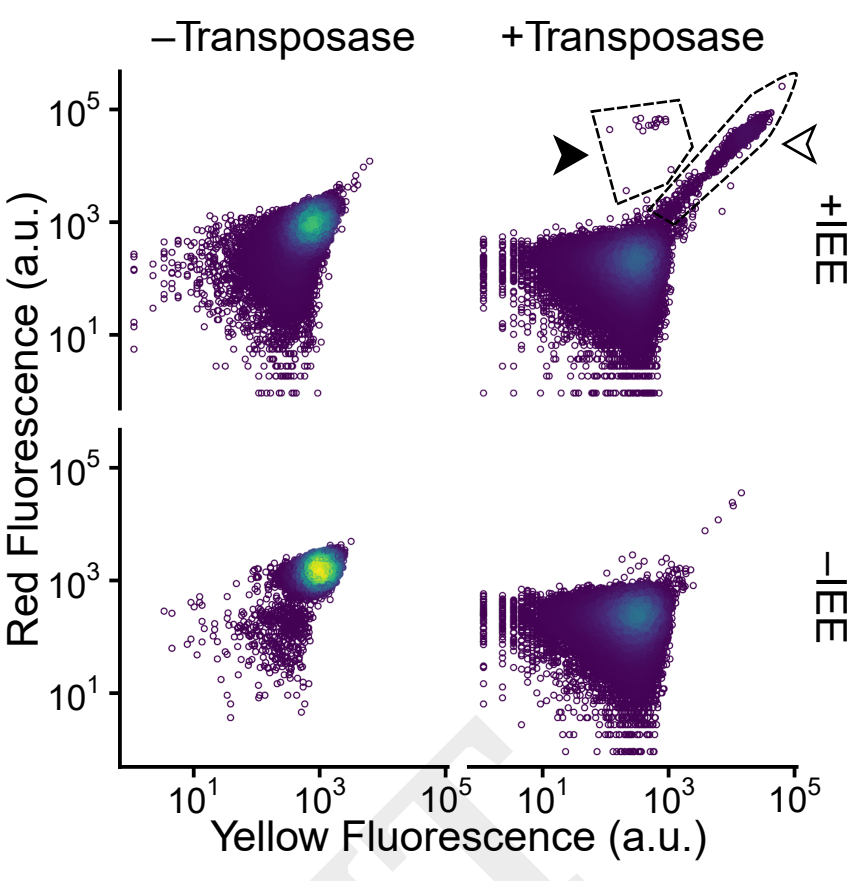

Figure 3. Single-cell fluorescence of cells with and without tpn and iee measured by flow cytometry $(n=50,000)$. The colors of the points indicate the density of points in the fraction; yellow indicates dense, and blue indicates sparse.

that with both tpn and iee, a large proportion of cells would show an increased level of yellow and red fluorescence by losing the copy of IS3. We also expected some cells to show an increase in only red fluorescence as IS 3 can disrupt $y f p$.

To test if the activity of IS and IEE creates the expected fluorescence patterns, cells transformed with the pairs of plasmids were cultured overnight with both iee and tpn fully induced (Figure 3, Supplementary Figure 1). We found that when both tpn and iee were present, a fraction of cells showed intense yellow and red fluorescence (Figure 3, white arrowhead). While these cells were also found in other conditions, they were most apparent when both tpn and iee were present and fully induced. To validate the importance of having both tpn and iee, Type III ANOVA was performed with the proportion of measurements with red fluorescence ten times brighter than the median of each measurement as the response variable and the presence of tpn, iee and the interaction of the two as the treatments. We found that the synergy of tpn and iee significantly facilitated the appearance of cells with intense fluorescence $\left(F_{1,12}=1.5 \times\right.$ $10^{-3}, P=1.0 \times 10^{-10}$, coefficient $\left.=3.8 \times 10^{-2}\right)$. Because the combined expression of tpn and iee has been associated with the excision of IS3 (25), this suggests that, in the bright cells, IS 3 was excised, and $y f p$ and $r f p$ were transcribed from the stronger promoter of kanR.

Besides cells showing both stronger yellow and red fluorescence, some cells with tpn and iee showed stronger red fluorescence but modest yellow fluorescence (Figure 3, black arrowhead). This implies that, as intended, not just the excision of IS 3 but also the deletion of adjacent $y f p$ may have occurred.

We also noticed that the intensity of fluorescence decreased 
with the expression of either or both of tpn and iee (Figure 3, Supplementary Figure 2.A). ANOVA with the median $\log$ fluorescence values as the response variable and the expression of tpn, iee, and the interaction of the two as treatments showed that the influence of tpn expression was the largest and most significant $\left(F_{1,12}=7.9 \times 10^{2}\right.$, $\left.P=2.6 \times 10^{-12}\right)$, corresponding to a 0.15 -fold decrease in intensity. The expression of iee reduced the intensity by 0.64 -fold, and the influence was also significant $\left(F_{1,12}=\right.$ $\left.4.3 \times 10, P=2.6 \times 10^{-5}\right)$. Nevertheless, no apparent growth defect was observed (Supplementary Figure 2.B), and the fluorescent protein genes of cells collected from the major fraction of the distributions were not deleted (Supplementary Figure 3). The unexpected decrease in fluorescence seems to indicate that, during operon formation, the activity of IS can alter the expression pattern of neighboring genes.

\section{IS generated a variety of genotypes, including those that seem to have formed operons}

To determine whether the altered fluorescence of the cells reflects the formation of novel operons, some cells with tpn and with or without iee were sorted based on their fluorescence (gates P1-3) by FACS (Figure 4.A). First, cells with both tpn and iee that evolved as four independent populations were analyzed. Consistent with Figure 3, a notable proportion of cells were found in P2 $(p=$ $10^{-1.3 \pm 0.1}, \mathrm{CI}$ ), and a few cells in P1 and P3 were also found with probabilities of $10^{-4.1 \pm 0.4}(\mathrm{CI})$ and $10^{-3.3 \pm 0.4}$ (CI), respectively. To investigate the diverse genotypes that seemed to have led to the formation of kanR-rfp operons, cells in P1 were collected from all four independent cultures. Cells in P2 and P3 were also collected from one population of cells. Next, a population of cells without the iee was also analyzed. Although the probability was negligible $(p<$ $10^{-5.5}$ ), cells in gate P1 were also collected for further analysis (Figure 4.B).

To assess if fluorescence was heritable, the cells were spread on LB agar plates with streptomycin and kanamycin and were regrown overnight. The fluorescence of colonies under blue/green LED was largely consistent with the fluorescence measured by FCM (Figure 4.C, Supplementary Figure 4). The cells from P1 showed red fluorescence as expected; cells from P2 and P3 generally showed green to orange fluorescence. However, some cells collected from P3 did not show bright fluorescence, suggesting that gate P3 contains populations overlapping with those in the major dark fraction of cells.

Next, sequences around the IS3 of the colonies were amplified by colony PCR to genotype the sorted cells. Fragments with deletions of typical lengths were chosen and sequenced by Sanger sequencing (Figure 4.D, Supplementary Table 2). The red colonies derived from cells with iee found in gate P1 showed deletions of sequences from the right inverted repeat (IRR) to $y f p$. In such cases, the plasmids tended to have the IS3 completely deleted. This result is in line with a study (25) that showed that IEE promotes the complete excision of IS3. The sequenced cells collected from gate P2 had the IS3 deleted up to the IRL. While many colonies had the IS3 completely deleted with excision starting adjacent to the IRR, some colonies had part of the IS3 remaining as in read b of Figure 4.D. Some cells collected from P3 had a genotype similar to those in P2, and others had only the sequence between IRL and $y f p$ deleted. The latter cells had the maximum possible deletion preserving the yellow fluorescence (read c, Supplementary Figure 5), implying that diverse types of deletions were generated by the activity of the IS. Overall, these results show that in line with previous studies on IS-mediated evolution $(21,25,30)$, the activity of IS causes deletions of various lengths, some of which form novel operons.

Rare cells in P1 without iee (Figure 4.B) also had $y f p$ deleted (Figure 4.D, P1 -IEE) (25). Among these cells, some cells also had the copy of IS3 excised as in read g of figure 4.D, but with parts of IS3 remained. This shows that although rarely, IS3 may also be able to form operons without IEE.

The deletions found by Sanger sequencing can be categorized into four types, as exemplified by reads a-d (Figure 4.E). When sequences between IS 3 and $r f p$ were deleted together with IS3, novel kanR-rfp operons formed (P1, read a). This type of deletion is consistent with the operon formation model proposed in this study. The excision of only IS3 led to the strong promoter of $k a n R$ to come closer to the $y f p$-rfp operon, forming $k a n R-y f p$-rfp operons ( $\mathrm{P} 2$, read b). Some cells did not form operons but had sequences adjacent to the IS3 deleted increasing red fluorescence. Deletion of sequences between IS 3 and $y f p$ led to stronger yellow fluorescence (P3, read c), and $y f p$ deletion led to the loss of yellow fluorescence (P1 -IEE, read d).

To demonstrate the diversity of operon-forming deletions IS can generate in the presence of iee, plasmids were extracted from cells sorted by FACS in P1. The deleted loci of the plasmids were briefly analyzed by nanopore sequencing. Consistent with the results of Sanger sequencing, deletions of various lengths were observed (Figure 4.F). The major types of deletions identified were those also found by Sanger sequencing of the colonies derived from the same population of cells collected by FACS (reads e and f). Although few, some reads had the IS3 sequence completely preserved, similar to read d. This implies that in some cells, transposase may have been excised the IS without IEE. In addition, similar to read b, some cells seemed to have IS3 partially left undeleted (25). These results are consistent with a previous study where some cells had intact or partially excised IS3, even when both IS3 and IEE were activated (25). Although sequencing by nanopore sequence is generally error-prone, the deletions found were largely consistent with those detected by Sanger sequencing, reinforcing that plasmids collected from the population of sorted cells contained a variety of deletions, many of which have led to operon formation. 

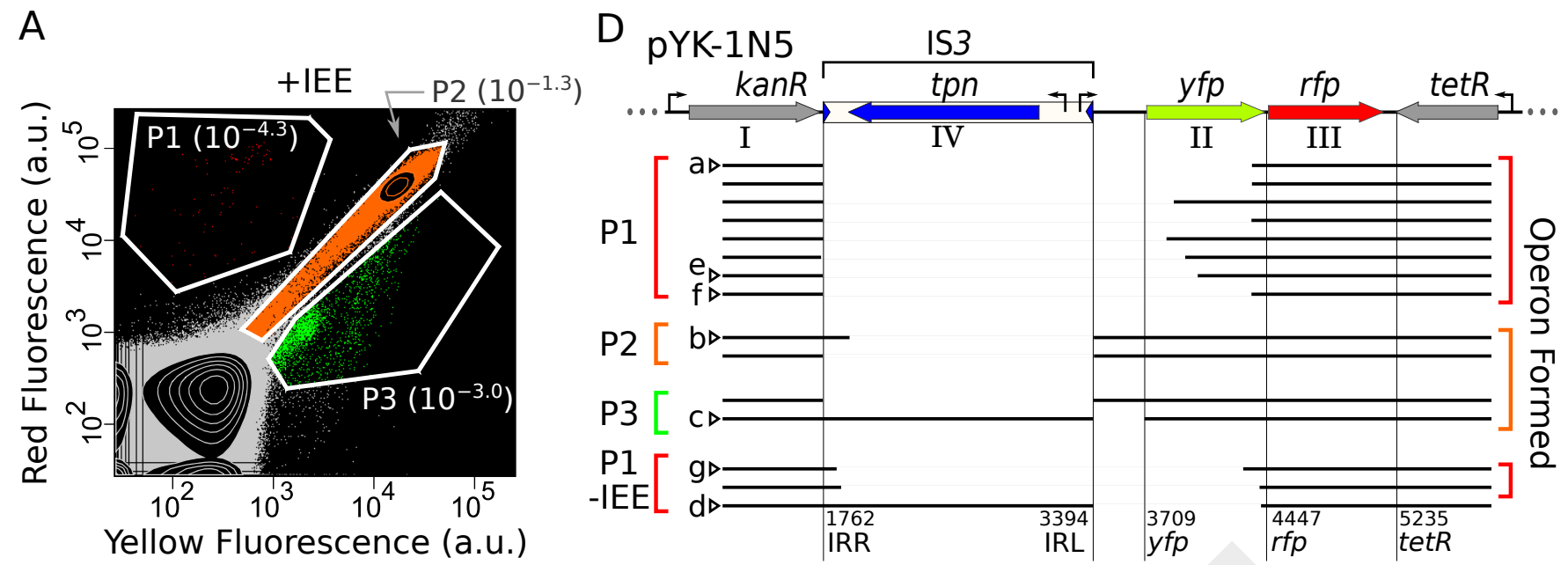

B

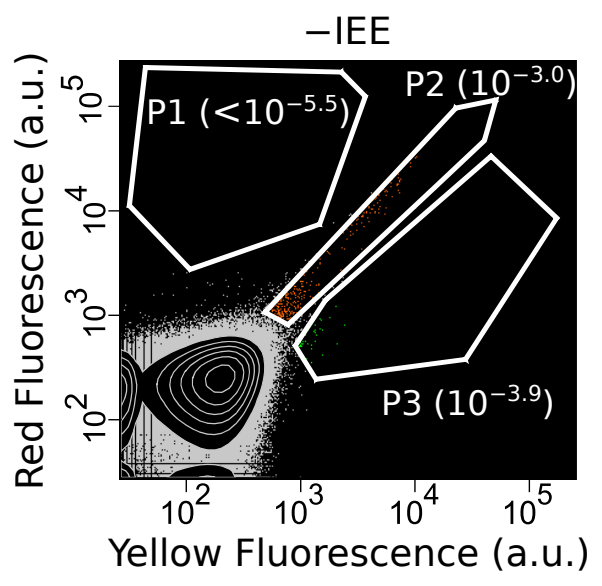

C

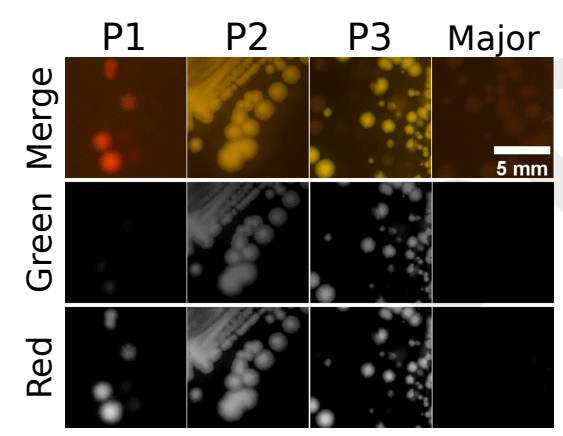

E Ancestor (yfp-rfp operon)
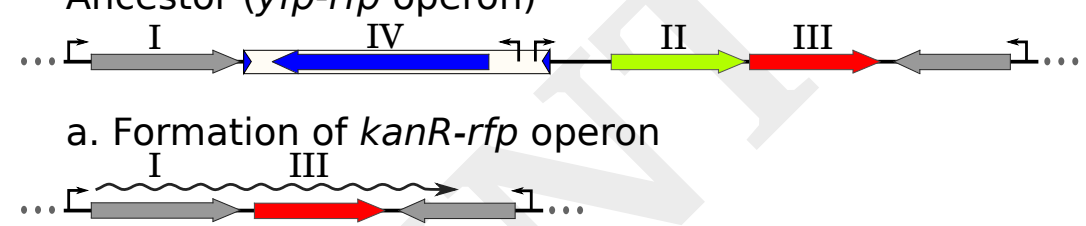

b. Formation of kanR-yfp-rfp operon

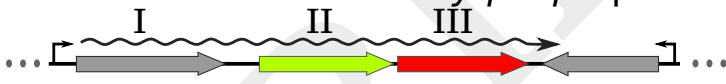

c. Stronger expression of $y f p$ and $r f p$

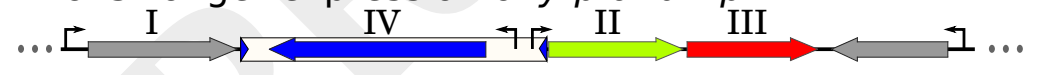

d. Stronger expression of $r f p$

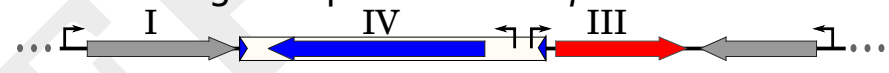

$\mathrm{F}$

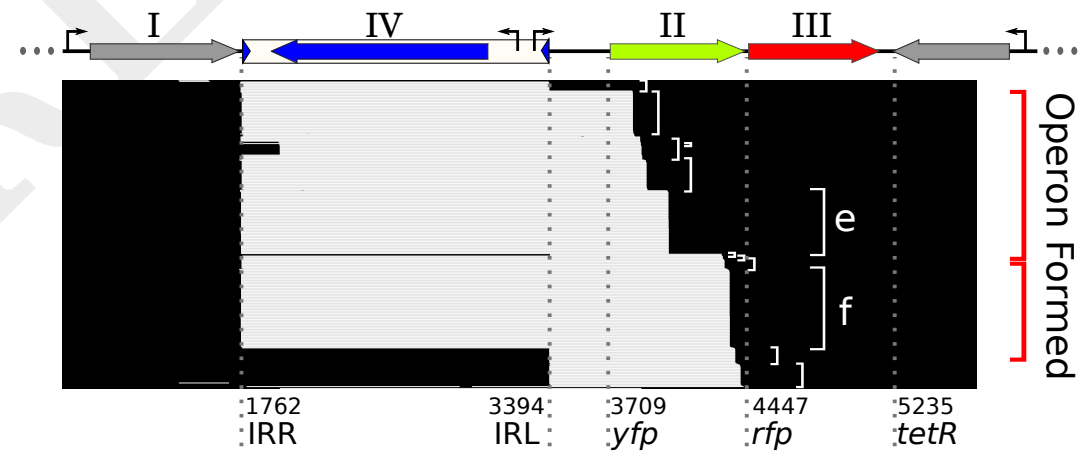

Figure 4. (A, B) Red and yellow fluorescence of cells with the IS3 transposase and with (A) or without (B) insertion sequence excision enhancer (IEE) expression vectors after evolution overnight ( $A: n=1,986,386 ; B: n=300,644)$. The transcription of both transposase and IEE genes (when present) were fully induced. Each polygon ( $P$ ) indicates the gates used to sort cells with fluorescence-activated cell sorting (FACS) for further analysis. The numbers within the parentheses show the proportion of cells found within each gate. No cell was found in gate P1 of subfigure B during this measurement. The cells were prepared under the same conditions as the measurements of Figure 2.C (A: +Transposase +IEE, B: +Transpsoase -IEE) but were from an independent population of cells. (C) Colonies of cells collected by FACS under blue/green LED. (D) Typical DNA sequences of pYK-1N5 that remained after evolution were determined by Sanger sequencing and are shown as black horizontal lines. The colors of the left square brackets correspond to the colors of points in subfigures A and B. The colors of the square brackets on the right correspond to the different types of operons formed; red indicates kanR-rfp operon, and orange indicates kanR-yfp-rfp operon. The vertical lines indicate the boundaries of genetic elements. The details of each deletion are provided in Supplementary Table 2. (E) The order of genes after the deletions shown in subfigure D. Each map corresponds to the sequence with the identical characters $(a-d)$ in subfigure D. (F) Analysis of the variation of deletions using nanopore sequencing. Plasmids extracted from a population of cells sorted from P1 in subfigure A by FACS were sequenced. The loci of deletions found in each read are shown as white horizontal lines. The white square brackets on the right indicate reads showing similar deletions. 


\section{Validation of the formation of novel operons}

To validate the formation of new operons, two experiments were performed.

First, the presence of mRNA transcribed from kanR to $r f p$ was detected by RT-PCR (Figure 5.A) using RNA extracted from cells corresponding to read e of Figure 4.D. The RNA was reverse transcribed into cDNA using the forward or reverse primer, and subsequently, the DNA was amplified using the pair of primers by PCR. We detected the expected 835 bp RNA only in the direction of kanR (Figure 5.B, e). This shows that the evolved cells had both kanR and $r f p$ on the same mRNA transcribed from the promoter of $k a n R$, validating the formation of a kanR-rfp operon. Using RNA extracted from cells corresponding to read $\mathrm{b}$ of Figure 4.D instead, we detected $1951 \mathrm{bp}$ RNA, validating the formation of a $k a n R-y f p-r f p$ operon (Figure 5.B, b).

Next, we tested whether the two genes could be controlled together. Specifically, we tested the coregulation of the two genes by replacing the promoter upstream of kanR with an IPTG inducible promoter $\mathrm{P}_{t a c}$ (Figure 5.A). We replaced the promoters of pYK-1N5 and its evolved derivatives and transformed the new plasmids into E. coli HST08 cells. The increase in the expression of $r f p$ with the addition of IPTG to the growth media was checked. We found that cells with plasmid reconstructed from the ancestor plasmid did not show increased red fluorescence. In contrast, cells with plasmids reconstructed using the evolved cells showed significantly stronger fluorescence with IPTG in the growth medium (Figure 5.C). Thus, deletions by the IS allowed the coregulation of $k a n R$ and $r f p$ as operons.

Overall, these experiments strongly support that cells evolved to have kanR and $r f p$ to be under the control of the same promoters.

\section{DISCUSSION}

\section{Summary of the results}

It is widely accepted that operon structures can change dramatically $(1,9,10,23,45)$. However, since the mechanism of operon formation has generally been studied by comparing genomes at the level above species, the very mutations that form new operons have been missed.

To explain the formation of operons, we proposed a new model whereby ISs facilitate the formation of novel operons through consecutive insertion-deletion-excision reactions (IDE model, Figure 1). According to this model, operons can form through intermediate adaptive steps (7). For experimental verification, we constructed a plasmid that mimics the first step of the proposed model (Figure 2), transformed it into an IS-less E. coli (Figure 3), and allowed it to evolve under the selection for kanamycin resistance and intense red fluorescence. We found that cells showed fluorescence indicative of IS-mediated deletions, especially when IEE was present (Figure 4). The structural mutations due to the transposition of IS caused rapid evolution that led

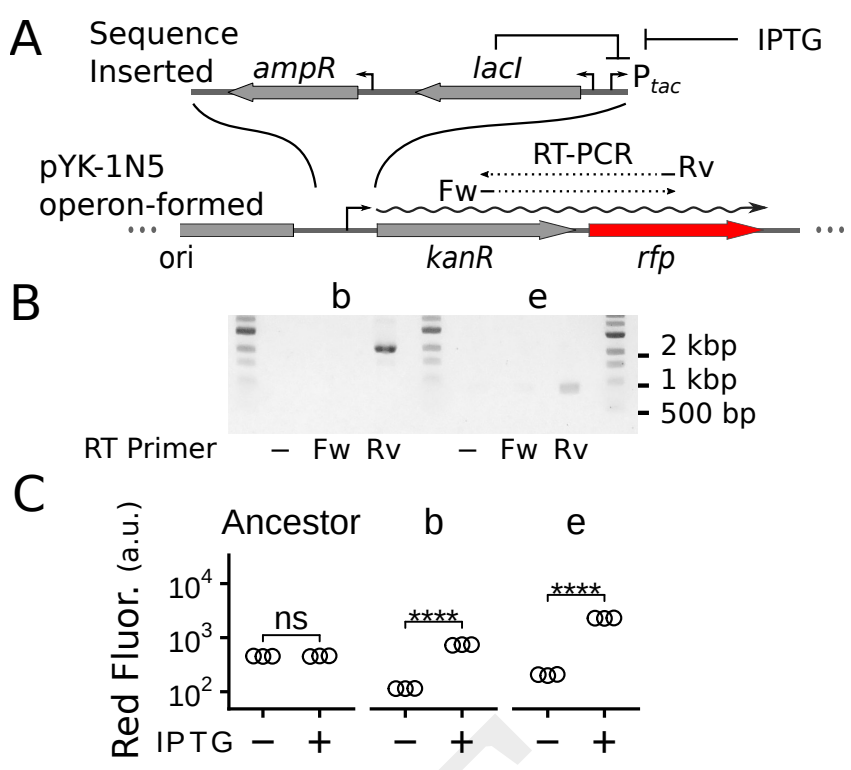

Figure 5. (A) The two experiments performed to confirm the formation of the kanRrfp operon. (B) Agarose gel electrophoresis of RT-PCR products. (C) Median red fluorescence measured by flow cytometry of cells with re-engineered plasmids $\left(^{* * * *}: p<10^{-4}\right)$. The constitutive promoter of kanR in pYK-1N5 was replaced with a DNA cassette containing an IPTG-inducible promoter $\left(\mathrm{P}_{\mathrm{tac}}\right)$. The lowercase letters indicate cells corresponding to reads in Figure 4.D with the same letter. Abbreviations: ampicillin resistance gene $(a m p R)$, lactose operon repressor gene (lacl), not significant (ns).

to the formation of novel operons (Figure 5) overnight in $200 \mu \mathrm{L}$ cultures.

\section{The IDE model is consistent with our understanding of the formation and maintenance of operons}

The IDE model is consistent with how operons in prokaryotes are thought to be under selection for better gene coregulation $(5,13-15,46)$ because adaptation for coregulation can begin once an operon has formed (7).

The IDE model is also consistent with our current understanding of how operons tend to form. For instance: (i) When new operons form, genes that are common across bacterial species are likely to be upstream of others (9), probably because this would preserve their sophisticated promoters even after deletion by ISs. (ii) When genes are added to existing operons, genes tend to be appended or prepended rather than inserted (9), probably because genes are added by ISs deleting redundant sequences.

\section{The advantages of the IDE model}

The IDE model is not only consistent with previous findings but is an efficient mechanism of operon formation from three perspectives.

(i) Gradual formation of operons The IDE model can incorporate the advantage of the 'selfish operon' model that states that operons can form without relying on rare events that directly cluster two genes into operons (7) (Figure 1.C). Even if we do not assume that higher expression of the two genes leads to higher fitness, selection for the loss of redundant sequences $(12,43,47)$ coupled with ISs that delete 
surrounding sequences can gradually bring two genes closer, forming a new operon.

(ii) Formation of suboptimal operons The expression levels of genes in operons are generally suboptimal (48), and genes in operons consisting of genes that are not functionally related tend to have functions important for cell growth (45). Our model is in line with these observations, as it explains operon formation without relying on the often-assumed selection for better gene coregulation. Consistently, various tendencies of operon formation, including the tendencies above, can be explained by assuming that operons form by selection for the preservation of genes rather than the selection for their coregulation $(9,12)$.

This explains an observation that inspired our new model, that is, several insect endosymbionts that have experienced extreme population bottlenecks (49) have small but operonrich genomes $(50,51)$. The selection for better coregulation would be too weak in these organisms for operons to form. Because insect symbionts generally reduce their genome size through a burst and a subsequent loss of ISs $(18,52)$, in line with our study, ISs may have formed their operon-rich genomes.

Our model improves the 'persistence model' (12), which currently relies upon the selection for coregulation to explain the formation of operons $(4,13)$. Unlike the 'persistence model,' we have attributed the large tandem deletions that cluster genes to the activity of ISs $(17,18)$. The activity of ISs alters the expression of surrounding genes (41). This enables cells with new IS insertions to become adaptive. In addition, this ensured that the premature loss of ISs after the deletion was maladaptive (Figure 1.C). It seems that the structure of ISs that have evolved for their proliferation may have made them a source of mutation particularly suitable for the formation of operons.

(iii) Accelerated recombination rate ISs not only actively transpose but are both drivers (53) and major targets of homologous recombination (54). They accelerate the formation of operons by transiently increasing the local structural mutation rates surrounding the IS $(17,30)$. How the increased mutation rate is kept local and transient is critical for our model because the rate of recombination can exceed the upper limit of global mutation rates set by the population size (55). In contrast, previous models based on random recombination ('recombination model' (11)) have been refuted because the rate of random recombination in prokaryotes seems to be too low to account for operon formation (7). Moreover, an experimental demonstration (Figure 4), unprecedented in any other models of operon formation, was possible because of the rapid mutation by IS3

The accelerated rate of recombination is crucial for the IDE model to act efficiently by overcoming the assumption that all genes present between two genes are dispensable (Figure 1.C). Recombination among multiple copies of IS enables multiple pairs of beneficial genes to be tested for undergoing the deletion step of operon formation. Furthermore, within prokaryotic genomes, some loci are easier to have only dispensable genes between two beneficial genes such as: pathogenicity islands (13), plasmids ('Scribbling Pad' hypothesis (56)), and clusters of nonessential genes $(12,16)$. ISs can actively form such loci by promoting recombination. For instance, duplication by IS-mediated recombination can create redundant copies of essential genes, making them nonessential ('SNAP hypothesis' (19)). In addition, recombination of two copies of ISs can form plasmids (24). Plasmid pYK-1N5 (Figure 2.A) can be formed from sequences within a chromosome by homologous recombination of a pair of IS3.

In conclusion, the operon-rich genomes of prokaryotes may have been shaped through the coexistence of ISs in the prokaryotic genome whereby operons rapidly form by the IDE model, newly-formed operons that are beneficial acquire sophisticated regulation and get fixed in genomes ('regulatory model' (5)), and organisms that have acquired operon-rich genomes become robust against the activity of ISs that cause large deletions ('persistence model' $(12,16))$.

\section{The activity of transposase may decrease the expres- sion of genes surrounding an IS}

An important characteristic of ISs is that they alter the expression of surrounding genes through their internal promoters (41). We observed that the increased activity of the IS3 transposase significantly decreased the expression of neighboring genes encoding fluorescent proteins (Figure 3). However, this did not seem to be due to the deletion of $r f p$ and $y f p$ (Supplementary Figure 4). Moreover, no growth defect was observed (Supplementary Figure 2.B), implying that the reduced copy number of plasmids due to their excision was not the cause because pYK-1N5 is a low copy number plasmid, and decreasing its copy number should have been detrimental to growth.

Rather, we speculate that the decreased fluorescence could have been due to the nicking of DNA by the transposase, the binding of transposase to the IRs, the formation of a protein DNA complex called 'Figure-eight' (40), or the increased transcription itself strengthening the supercoiling of DNA (57). While solving the dominant cause of the decreased fluorescence is out of our scope, we speculate that ISs may act as global regulators, with IS transposase acting as transcription repressors and IS inverted repeats as operator sequences. Supporting this, ISs have been found in orientations that interfere with neighboring genes in clinical samples of Staphylococcus aureus (58).

\section{Limitations of our study}

Our model is limited in that some operons are likely to have formed using mechanisms other than that proposed by our model. For instance, new operons can form by replacing the genes of preexisting operons (9) or by duplication (10). Also, toxin-antitoxin systems form operons, probably by the 'selfish operon' model. 
Second, although our experiment shows that ISs cause diverse deletions that can form operons, the process of operon formation may likely be much slower in nature without the enhanced activity of the IS and IEE. Consistently, in our experimental demonstration, the rate of operon formation was much lower when IEE was not present (Figure 4.B, P1). Nevertheless, as our observations were largely consistent with those of previous studies, we believe that the results are robust to minor changes in the design of the experimental model.

Finally, while deletion and excision of IS is common in major types of ISs $(20,22)$, we only demonstrated the formation of operons using the combination of IS3 and IEE in a strain of E. coli. We believe, to extend the scope of our model, it is necessary to understand the genetic factors essential for the insertion, deletion, and excision reactions by IS transposase and the types of ISs that promote operon formation. Future studies in such directions might also reveal the origins of ancient operons $(10,19)$ by comparing when the genetic factors, the ISs, and the operons emerged.

\section{Future work}

Our study is probably the first experimental demonstration of operon formation in the laboratory. However, the proposed model requires validation in nature. This is important as ISs are regarded as drivers of operon destruction $(19,23)$, although this view may be biased because studies have focused more on the evolution of conserved operons than on their formation.

A potential difficulty to find examples of operon formation in nature is that IS can be thought of as a genetic "catalyst" that locally accelerates the mutation rate and transforms a genome into a new genome with an additional operon (Figure 1.C). As a catalyst, the activity of IS is virtually traceless. As a result, previous studies have overlooked the role of deletions by IS in operon formation because, based on parsimony of events, operon formation by the IDE model cannot be distinguished from large tandem deletions without ISs (9). Indeed, partial sequences of ISs may remain as read $\mathrm{g}$ of Figure 4.D, but these sequences would be rapidly lost, as they are unlikely to have any function. We believe that to find cases where operons are formed by our model and to determine whether ISs are the major drivers of operon formation, analyzing ongoing evolutions in detail $(28,54,59,60)$ to detect the intermediate steps of operon formation is essential.

We postulate that these steps may potentially be identified in organisms that are under reductive evolution (61), which ISs are known to facilitate $(18,43)$. When genomes expand, ISs can potentiate newly acquired genes to form operons by activating them. As genome size decreases, ISs delete redundant sequences and excise themselves, forming new operons. Supporting this idea, a systematic study of genome size evolution in cyanobacteria showed that operon-rich genomes tend to have experienced genome reduction (14). In contrast, larger genomes with many ISs are relatively poor in operons (4), perhaps because they are yet to experience an upsurge in operon formation. Future studies on, for example, the evolution of pathogenic E. coli with multiple ISs that degrade their genomes (62) and are excised by IEE (63), or organisms with genome size evolution artificially accelerated using hyper-active transposons (64) may provide a clearer picture of the coevolution of prokaryotic genomic and regulatory architecture with ISs, as suggested by our study.

\section{DATA AVAILABILITY}

Nanopore sequencing data have been deposited with SRA (BioProject PRJNA768397).

The raw flow cytometry data are available in FlowRepository (FR-FCM-Z4LV).

\section{SUPPLEMENTARY DATA}

Supplementary Data are available at NAR Online: Supplementary Figures 1-6, Supplementary Tables 1-2, and DNA sequences.

\section{AUTHOR CONTRIBUTIONS}

Conceptualization and methodology: Y.K. and S.T.; data curation, formal analysis, investigation, validation, and visualization: Y.K.; funding acquisition: Y.K., S.T., and C.F.; project administration: S.T.; resources and supervision: S.T. and C.F.; writing - original draft: Y.K.; writing - review \& editing: Y.K., S.T., and C.F.

\section{ACKNOWLEDGMENTS}

The authors thank the following people for generously sharing their reagents: The mScarlet-I sequence was generated by mutating the mScarlet sequence, which was a gift from Dr. Ryudo Ohbayashi. The Tn5 transposase sequence used as -tpn control was a gift from Dr. Ronald Chalmers. The Marionette Sensor Collection was a gift from Dr. Christopher Voigt (Addgene \#1000000137). pCDSSaraL18 was a gift from Dr. Peter Schultz.

\section{FUNDING}

This work was supported by the Japan Society for the Promotion of Science [21J20693 to Y.K., 18H02427 to S.T., 17 H06389 to C.F, 19 H05626 to C.F]; and the Japan Science and Technology Agency [JPMJER1902 to C.F]. Funding for open access charge: the Japan Science and Technology Agency.

\section{Conflict of interest statement. None declared.}

\footnotetext{
1. Wolf, Y. I., Rogozin, I. B., Kondrashov, A. S., and Koonin, E. V. (2001) Genome alignment, evolution of prokaryotic genome organization, and prediction of gene function using genomic context. Genome Res., 11(3), 356-372.

2. Koonin, E. V. and Wolf, Y. I. (2008) Genomics of bacteria and archaea: the emerging dynamic view of the prokaryotic world. Nucleic Acids Res., 36(21), 6688-6719.

3. Jacob, F. and Monod, J. (1961) Genetic regulatory mechanisms in the synthesis of proteins. J. Mol. Biol., 3(3), 318-356.

4. Nuñez, P. A., Romero, H., Farber, M. D., and Rocha, E. P. (2013) Natural selection for operons depends on genome size. Genome Biol. Evol., 5(11), 2242-2254.

5. Price, M. N., Huang, K. H., Arkin, A. P., and Alm, E. J. (2005) Operon formation is driven by co-regulation and not by horizontal gene transfer. Genome Res., 15(6), 809-819.
} 
6. Sneppen, K., Pedersen, S., Krishna, S., Dodd, I., and Semsey, S. (2010) Economy of operon formation: Cotranscription minimizes shortfall in protein complexes. MBio, 1(4), 4-6.

7. Lawrence, J. G. and Roth, J. R. (1996) Selfish operons: Horizontal transfer may drive the evolution of gene clusters. Genetics, 143(4), 1843-1860.

8. Shieh, Y. W., Minguez, P., Bork, P., Auburger, J. J., Guilbride, D. L., Kramer, G., and Bukau, B. (2015) Operon structure and cotranslational subunit association direct protein assembly in bacteria. Science (80-. )., 350(6261), 678-680.

9. Price, M. N., Arkin, A. P., and Alm, E. J. (2006) The life-cycle of operons. PLoS Genet., 2(6), 0859-0873.

10. Fani, R., Brilli, M., and Liò, P. (2005) The origin and evolution of operons: the piecewise building of the proteobacterial histidine operon. J. Mol. Evol., 60(3), 378-390.

11. Stahl, F. W. and Murray, N. E. (1966) The evolution of gene clusters and genetic circularity in microorganisms. Genetics, 53(3), 569-576.

12. Fang, G., Rocha, E. P., and Danchin, A. (2008) Persistence drives gene clustering in bacterial genomes. BMC Genomics, 9.

13. Touchon, M. and Rocha, E. P. (2016) Coevolution of the organization and structure of prokaryotic genomes. Cold Spring Harb. Perspect. Biol., 8(1), a018168.

14. Memon, D., Singh, A. K., Pakrasi, H. B., and Wangikar, P. P. (2013) A global analysis of adaptive evolution of operons in cyanobacteria. Antonie Van Leeuwenhoek, 103(2), 331346.

15. Pál, C. and Hurst, L. D. (2004) Evidence against the selfish operon theory. Trends Genet., 20(6), 232-234.

16. Hosseini, S. R. and Wagner, A. (2018) Genomic organization underlying deletional robustness in bacterial metabolic systems. Proc. Natl. Acad. Sci. U. S. A., 115(27), 70757080.

17. Lee, H., Doak, T. G., Popodi, E., Foster, P. L., and Tang, H. (2016) Insertion sequencecaused large-scale rearrangements in the genome of Escherichia coli. Nucleic Acids Res., 44(15), 7109-19.

18. Siguier, P., Gourbeyre, E., and Chandler, M. (2014) Bacterial insertion sequences: their genomic impact and diversity. FEMS Microbiol. Rev., 38(5), 865-891.

19. Brandis, G. (2021) Reconstructing the evolutionary history of a highly conserved operon cluster in Gammaproteobacteria and Bacilli. Genome Biol. Evol., 13(4).

20. Reif, H. J. and Saedler, H. (1975) IS 1 is involved in deletion formation in the gal region of $E$. coli K12. Mol. Gen. Genet., 137(1), 17-28.

21. Sekine, Y., Eisaki, N., and Ohtsubo, E. (1994) Translational control in production of transposase and in transposition of insertion sequence IS3. J. Mol. Biol., 235(5), 14061420.

22. Jilk, R. A., Makris, J. C., Borchardt, L., and Reznikoff, W. S. (1993) Implications of Tn5associated adjacent deletions. J. Bacteriol., 175(5), 1264-1271.

23. Itoh, T., Takemoto, K., Mori, H., and Gojobori, T. (1999) Evolutionary instability of operon structures disclosed by sequence comparisons of complete microbial genomes. Mol. Biol. Evol., 16(3), 332-346.

24. Snyder, L., M, H. T., Peters, J. E., and Champness, W. (2013) Molecular genetics of bacteria, ASM Press, Washington, D.C.

25. Kusumoto, M., Ooka, T., Nishiya, Y., Ogura, Y., Saito, T., Sekine, Y., Iwata, T., Akiba, M., and Hayashi, T. (2011) Insertion sequence-excision enhancer removes transposable elements from bacterial genomes and induces various genomic deletions. Nat. Commun., 2, 152.

26. Price, M. N., Huang, K. H., Alm, E. J., and Arkin, A. P. (2005) A novel method for accurate operon predictions in all sequenced prokaryotes. Nucleic Acids Res., 33(3), 880-892.

27. Chayot, R., Montagne, B., Mazel, D., and Ricchetti, M. (2010) An end-joining repair mechanism in Escherichia coli. Proc. Natl. Acad. Sci., 107(5), 2141-2146.

28. Ooka, T., Ogura, Y., Asadulghani, M., Ohnishi, M., Nakayama, K., Terajima, J., Watanabe, H., and Hayashi, T. (2009) Inference of the impact of insertion sequence (IS) elements on bacterial genome diversification through analysis of small-size structural polymorphisms in Escherichia coli 0157 genomes. Genome Res., 19(10), 1809-1816.

29. Chain, P. S., Hu, P., Malfatti, S. A., Radnedge, L., Larimer, F., Vergez, L. M., Worsham, P., Chu, M. C., and Andersen, G. L. (2006) Complete genome sequence of Yersinia pestis strains antiqua and Nepal516: Evidence of gene reduction in an emerging pathogen. $J$. Bacteriol., 188(12), 4453-4463.

30. Cooper, V. S., Schneider, D., Blot, M., and Lenski, R. E. (2001) Mechanisms causing rapid and parallel losses of ribose catabolism in evolving populations of Escherichia coli B. J. Bacteriol., 183(9), 2834-2841.

31. Pósfai, G., Plunkett, G., Fehér, T., Frisch, D., Keil, G. M., Umenhoffer, K., Kolisnychenko, V., Stahl, B., Sharma, S. S., de Arruda, M., Burland, V., Harcum, S. W., and Blattner, F. R. (2006) Emergent properties of reduced-genome Escherichia coli. Science (80-. )., 312(5776), 1044-1046.

32. Shoji, S., Dambacher, C. M., Shajani, Z., Williamson, J. R., and Schultz, P. G. (2011) Systematic chromosomal deletion of bacterial ribosomal protein genes. J. Mol. Biol., 413(4), 751-761.

33. Blundell-Hunter, G., Tellier, M., and Chalmers, R. (2018) Transposase subunit architecture and its relationship to genome size and the rate of transposition in prokaryotes and eukaryotes. Nucleic Acids Res., 46(18), 9637-9646.

34. Bindels, D. S., Haarbosch, L., Van Weeren, L., Postma, M., Wiese, K. E., Mastop, M., Aumonier, S., Gotthard, G., Royant, A., Hink, M. A., and Gadella, T. W. (2016) mScarlet: A bright monomeric red fluorescent protein for cellular imaging. Nat. Methods, 14(1), 53-56.

35. Nagai, T., Ibata, K., Park, E. S., Kubota, M., Mikoshiba, K., and Miyawaki, A. (2002) A variant of yellow fluorescent protein with fast and efficient maturation for cell-biological applications. Nat. Biotechnol., 20, 87-90.

36. Lutz, R. and Bujard, H. (1997) Independent and tight regulation of transcriptional units in Escherichia coli via the LacR/O, the TetR/O and $\mathrm{AraC} / \mathrm{I}_{1}-\mathrm{I}_{2}$ regulatory elements. Nucleic Acids Res., 25(6), 1203-1210.

37. Meyer, A. J., Segall-Shapiro, T. H., Glassey, E., Zhang, J., and Voigt, C. A. (2019) Escherichia coli "Marionette" strains with 12 highly optimized small-molecule sensors. Nat. Chem. Biol., 15(2), 196-204.

38. Silander, O. K., Nikolic, N., Zaslaver, A., Bren, A., Kikoin, I., Alon, U., and Ackermann, M. (2012) A genome-wide analysis of promoter-mediated phenotypic noise in Escherichia coli.
PLoS Genet., 8(1), e1002443

39. Li, H. (2018) Minimap2: Pairwise alignment for nucleotide sequences. Bioinformatics, 34(18), 3094-3100.

40. Chandler, M., Fayet, O., Rousseau, P., Hoang, B. T., and Duval-valentin, G. (2015) Copyout-paste-in transposition of IS911: a major transposition pathway. Microbiol Spectr., 3(4), MDNA3-0031-2014.

41. Vandecraen, J., Chandler, M., Aertsen, A., and Van Houdt, R. (2017) The impact of insertion sequences on bacterial genome plasticity and adaptability. Crit. Rev. Microbiol., 43(6), 709730 .

42. Charlier, D., Piette, J., and Glansdorff, N. (1982) IS3 can function as a mobile promoter in E. coli. Nucleic Acids Res., 10(19), 5935-5948.

43. van Dijk, B., Bertels, F., Stolk, L., Takeuchi, N., and Rainey, P. B. (2021) Transposable elements drive the evolution of genome streamlining. bioRxiv doi: https://doi.org/10.1101/2021.05.29.446280, 29 May 2021, pre-print: not peer-reviewed.

44. Fehér, T., Bogos, B., Méhi, O., Fekete, G., Csörgő, B., Kovács, K., Pósfai, G., Papp, B., Hurst, L. D., and Pál, C. (2012) Competition between transposable elements and mutator genes in bacteria. Mol. Biol. Evol., 29(10), 3153-3159.

45. Rogozin, I. B., Makarova, K. S., Murvai, J., Czabarka, E., Wolf, Y. I., Tatusov, R. L., Szekely, L. A., and Koonin, E. V. (2002) Connected gene neighborhoods in prokaryotic genomes. Nucleic Acids Res., 30(10), 2212-2223.

46. Lim, H. N., Lee, Y., and Hussein, R. (2011) Fundamental relationship between operon organization and gene expression. Proc. Natl. Acad. Sci. U. S. A., 108(26), 10626-10631.

47. Koskiniemi, S., Sun, S., Berg, O. G., and Andersson, D. I. (2012) Selection-driven gene loss in bacteria. PLoS Genet., 8(6), e1002787.

48. Price, M. N., Deutschbauer, A. M., Skerker, J. M., Wetmore, K. M., Ruths, T., Mar, J. S., Kuehl, J. V., Shao, W., and Arkin, A. P. (2013) Indirect and suboptimal control of gene expression is widespread in bacteria. Mol. Syst. Biol., 9(660), 1-18.

49. Moran, N. A. (2002) Microbial minimalism: Genome reduction in bacterial pathogens. Cell, $108(5), 583-586$

50. Brinza, L., Calevro, F., Duport, G., Gaget, K., Gautier, C., and Charles, H. (2010) Structure and dynamics of the operon map of Buchnera aphidicola sp. strain APS. BMC Genomics, 11(1).

51. Güell, M., van Noort, V., Yus, E., Chen, W.-H., Leigh-Bell, J., Michalodimitrakis, K., Yamada, T., Arumugam, M., Doerks, T., Kühner, S., Rode, M., Suyama, M., Schmidt, S., Gavin, A.-C., Bork, P., and Serrano, L. (2009) Transcriptome complexity in a genome-reduced bacterium. Science (80-. )., 326(5957), 1268-1271.

52. McCutcheon, J. P. and Moran, N. A. (2012) Extreme genome reduction in symbiotic bacteria. Nat. Rev. Microbiol., 10, 13-26.

53. Reams, A. B., Kofoid, E., Kugelberg, E., and Roth, J. R. (2012) Multiple pathways of duplication formation with and without recombination (RecA) in Salmonella enterica. Genetics, 192(2), 397-415.

54. Raeside, C., Gaffé, J., Deatherage, D. E., Tenaillon, O., Briska, A. M., Ptashkin, R. N., Cruveiller, S., Médigue, C., Lenski, R. E., Barrick, J. E., and Schneider, D. (2014) Large chromosomal rearrangements during a long-term evolution experiment with Escherichia coli. MBio, 5(5), e01377-14.

55. Lynch, M., Ackerman, M. S., Gout, J. F., Long, H., Sung, W., Thomas, W. K., and Foster, P. L. (2016) Genetic drift, selection and the evolution of the mutation rate. Nat. Rev. Genet., 17(11), 704-714.

56. Norris, V. and Merieau, A. (2013) Plasmids as scribbling pads for operon formation and propagation. Res. Microbiol., 164(7), 779-787.

57. Yeung, E., Dy, A. J., Martin, K. B., Ng, A. H., Del Vecchio, D., Beck, J. L., Collins, J. J., and Murray, R. M. (2017) Biophysical constraints arising from compositional context in synthetic gene networks. Cell Syst., 5(1), 11-24.

58. Yebra, G., Haag, A. F., Neamah, M. M., Wee, B. A., Richardson, E. J., Horcajo, P., Granneman, S., Tormo-Más, M. Á., de la Fuente, R., Fitzgerald, J. R., and Penadés, J. R. (2021) Radical genome remodelling accompanied the emergence of a novel host-restricted bacterial pathogen. PLOS Pathog., 17(5), e1009606.

59. Consuegra, J., Gaffé, J., Lenski, R. E., Hindré, T., Barrick, J. E., Tenaillon, O., and Schneider, D. (2021) Insertion-sequence-mediated mutations both promote and constrain evolvability during a long-term experiment with bacteria. Nat. Commun., 12, 980.

60. He, S., Chandler, M., Varani, A. M., Hickman, A. B., Dekker, J. P., and Dyda, F. (2016) Mechanisms of evolution in high-consequence drug resistance plasmids. MBio, 7(6), 1-11.

61. Wolf, Y. I. and Koonin, E. V. (2013) Genome reduction as the dominant mode of evolution. BioEssays, 35(9), 829-837.

62. Hawkey, J., Monk, J. M., Billman-Jacobe, H., Palsson, B., and Holt, K. E. (2020) Impact of insertion sequences on convergent evolution of Shigella species. PLOS Genet., 16(7), 1-32.

63. Toro, M., Rump, L. V., Cao, G., Meng, J., Brown, E. W., and Gonzalez-Escalona, N. (2015) Simultaneous presence of insertion sequence excision enhancer and insertion sequence IS629 correlates with increased diversity and virulence in Shiga toxin-producing Escherichia coli. J. Clin. Microbiol., 53(11), 3466-3473.

64. Vernyik, V., Karcagi, I., Tímár, E., Nagy, I., Györkei, Á., Papp, B., Györfy, Z., and Pósfai, G. (2020) Exploring the fitness benefits of genome reduction in Escherichia coli by a selectiondriven approach. Sci. Rep., 10, 7345 